\title{
Extraintestinal Seeding of Salmonella enterica Serotype Typhi, Pakistan
}

Seema Irfan, Mohammad Zeeshan, Salima Rattani, Joveria Farooqi, Sadia Shakoor, Rumina Hasan, Afia Zafar

We evaluated Salmonella enterica serotype Typhi strains isolated from all body sites in Pakistan during 2013-2018. Despite an increase in overall number of localized, extensively drug-resistant Salmonella Typhi in organ infections during 2018, there was no increase in the proportion of such isolates in comparison with non-extensively drugresistant isolates.

Calmonella enterica serotype Typhi is a major patho$\checkmark$ gen affecting populations from low- and middleincome countries that generally lack clean, potable water and good sanitary disposal systems (1). The global incidence of enteric fever is $\approx 21$ million cases annually, and there are $\approx 200,000$ typhoid-related deaths/year (2). Pakistan is among the high-burden countries that has reported annual incidence of 493.5 case/100,000 persons (3-5). Seeding of deep-seated organs by Salmonella Typhi, resulting in bone and soft tissue infections and splenic and hepatic abscesses, has been reported (6-9). Extensively drug-resistant (XDR) Salmonella Typhi, a strain resistant to 5 groups of antimicrobial drugs, including third-generation cephalosporins $(10,11)$, has emerged in 2 cities in the southern part of Sindh Province and further disseminated to other parts of Pakistan, raising concern for persistence of the organism in hosts because of delays in appropriate therapy.

During 2018, a sudden increase in isolation frequency of XDR Salmonella Typhi from clinical samples other than blood, stool, and urine in Pakistan was observed. We conducted a study to determine if there was a true increase in the proportion of extraintestinal XDR Salmonella Typhi infections compared with non-XDR infections.

\section{The Study}

This study was conducted at the clinical microbiology laboratory at Aga Khan Hospital (Karachi, Pakistan).

Author affiliation: Aga Khan University, Karachi, Pakistan

DOI: https://doi.org/10.3201/eid2703.200464
After approval was obtained from the Ethical Review Committee at Aga Khan Hospital, all reports of clinical specimens that showed growth of Salmonella Typhi during January 2013-December 2018 were extracted from the laboratory database and included in the study. The frequency of isolation of the organism from extraintestinal organ infections was compared with that of blood/bone marrow, stool, and urine. In addition, informed consent and detailed history were obtained by telephone from patients who had XDR Salmonella Typhi isolated from sites other than blood/bone marrow, stool, and urine during 2018.

Salmonella Typhi were identified by using conventional biochemical reactions and API 20E (bioMérieux, https://www.biomerieux.com) and then confirmed by serotyping with Salmonella antisera (Becton Dickinson, https://www.bd.com). Susceptibility testing was performed by using the disk diffusion Kirby-Bauer method and recent Clinical and Laboratory Standards Institute (https:/ / clsi.org) performance standards. For XDR Salmonella Typhi strains, susceptibility was confirmed by using the Vitek2 System (bioMérieux), except for azithromycin, which was reported by using the disk diffusion method. The Pearson $\chi^{2}$ test was applied to calculate the statistical significance by using Stata SE 12.1 software (https:/ / www.stata.com).

During the 6-year study period, 8,736 isolates of Salmonella Typhi were reported from blood, bone marrow, stool, and urine, and 62 isolates were reported from other body sites (Table 1). Yearly isolation of Salmonella Typhi from different body sites gradually decreased during 2013-2017, but during 2018, there was a slight increase. In addition, although XDR Salmonella Typhi isolation from blood, feces, and urine had been consistently increasing since the beginning of outbreak, its isolation from other body sites was not observed until 2017. During 2018, these strains emerged from other sterile body tissues and fluids. However, their isolation 
Extraintestinal Seeding of Salmonella Typhi

Table 1. Salmonella enterica serotype Typhi isolates from blood, feces, and urine versus other body sites, Pakistan 2013-2018

\begin{tabular}{|c|c|c|c|c|c|}
\hline \multicolumn{2}{|c|}{ Characteristic } & $\begin{array}{c}\text { No. (\%, } 95 \% \mathrm{Cl}) \text { in blood, } \\
\text { feces, or urine }\end{array}$ & $\begin{array}{c}\text { No. }(\%, 95 \% \mathrm{Cl}) \text { in other body } \\
\text { sites }\end{array}$ & Total cases & $\mathrm{p}$ value \\
\hline \multicolumn{2}{|c|}{ No. ceftriaxone sensitive } & $5,858(99.1,98.9-99.4)$ & $51(0.86,0.63-1.10)$ & 5,909 & 0.011 \\
\hline \multicolumn{2}{|c|}{ No. ceftriaxone resistant } & $2,878(99.6,99.4-99.8)$ & $11(0.38,0.16-0.61)$ & 2,889 & \\
\hline \multicolumn{2}{|c|}{ Total } & $8,736(99.3,99.1-99.5)$ & $62(0.70,0.53-0.88)$ & 8,798 & \\
\hline \multicolumn{6}{|c|}{ Ceftriaoxne susceptibility } \\
\hline \multirow[t]{2}{*}{2013} & Sensitive & $662(98.5,97.6-99.4)$ & $10(1.49,0.57-2.41)$ & 672 & 0.902 \\
\hline & Resistant & 0 & 0 & 0 & \\
\hline \multirow[t]{2}{*}{2014} & Sensitive & $701(98.7,97.9-99.6)$ & $9(1.27,0.45-2.10)$ & 710 & 0.873 \\
\hline & Resistant & 0 & 0 & 0 & \\
\hline \multirow[t]{2}{*}{2015} & Sensitive & $881(98.9,98.3-99.6)$ & $9(1.01,0.36-1.68)$ & 890 & 0.839 \\
\hline & Resistant & 0 & 0 & 0 & \\
\hline \multirow[t]{2}{*}{2016} & Sensitive & 1,167 (99.5, 99.2-99.9) & $5(0.42,0.05-0.80)$ & 1,172 & 0.764 \\
\hline & Resistant & $21(100)^{*}$ & 0 & 21 & \\
\hline \multirow[t]{2}{*}{2017} & Sensitive & 1,299 (99.3, 98.9-99.8) & 9 (0.69) 24-1.14) & 1,308 & 0.057 \\
\hline & Resistant & $526(100)^{*}$ & 0 & 526 & \\
\hline \multirow[t]{2}{*}{$2018 \dagger$} & Sensitive & $1,155(99.2,98.7-99.7)$ & $9(0.77,0.27-1.28)$ & 1,164 & 0.264 \\
\hline & Resistant & 2,324 (99.5, 99.3-99.8) & $11(0.47,0.19-0.75)$ & 2,335 & \\
\hline
\end{tabular}

proportion was not significant compared with nonXDR isolates (Table 1).

Most (7/11, 63.6\%) cases showing growth of XDR Salmonella Typhi from other body sites were in children, but $4(36.4 \%)$ were in adults. The average duration from fever onset until care was sought was $\approx 2$ weeks. For $9(81.8 \%)$ of 11 case-patients, complications came in the form of deep abscesses at body sites such as gluteal muscles, deltoid muscles, spleen, subdiaphragmatic recess, pleural cavity, breast, and fractured site of a limb. One case-patient who had no concurrent conditions had acute meningitis, and another case-patient had acute abdominal pain secondary to intestinal perforation and leading to pneumoperitoneum.

Antimicrobial drugs such as clindamycin, ciprofloxacin, amoxicillin/clavulanic acid, ceftriaxone, and cefepime showed inappropriate coverage and treatment duration against XDR Salmonella Typhi and were used for $8 / 11(72.7 \%)$ patients during the course of illness. One patient was lost to follow up, but 10/11 (90.9\%) patients showed marked improvement after appropriate antimicrobial drug treatment (intramuscular meropenem or oral azithromycin) and survived (Table 2, https://wwwnc.cdc.gov/EID/ article/27/3/20-0464-T2.htm).

\section{Conclusions}

Our study was a comparison of isolation rates of XDR and non-XDR Salmonella Typhi from extraintestinal organ infections after the recognition of an XDR Salmonella Typhi outbreak in Pakistan. Our laboratory data highlights that, although isolation of XDR Salmonella Typhi from blood cultures has been performed since 2016, emergence and detection of XDR Salmonella Typhi from other body sites started during
2018, indicating a lag period of 14 months between the outbreak and extraintestinal organ infections. Except for 1 case of postileal perforation and collection of intraabdominal material, most of these manifestations were probably secondary to bacteremic seeding at various body sites. According to disease pathogenesis, different factors can contribute toward typhoid complication, including host-related factors, such as defects in innate immunity, any existing scar in soft tissue, inappropriate use of antimicrobial drugs, and organism-related virulence factors (12).

In this study, apparent immune dysfunction was found in only 2 patients: a 45 -year-old woman who had a breast abscess and diabetes mellitus, and a 15-year-old boy who had splenic abscess and autoimmune hepatitis. A 55-year-old patient had a history of splenic injury and later showed development of a splenic abscess. Inappropriate antimicrobial drug use (Table 2), a common finding for most uncomplicated enteric fever cases, could be another contributing factor for uncontrolled disease process, leading to complications. However, this factor cannot solely be identified as a risk factor for these complicated cases. Another finding was that 4 of 11 extraintestinal organ infections were in adults, despite enteric fever being a disease with highest occurrence among children. We suggest future cohort studies to determine the reason for this delay in appearance and distribution of complicated cases.

Our data showed that during 2013-2018, irrespective of susceptibility pattern, the proportion of complicated enteric fever cases in Pakistan decreased (Table 1). Nonetheless, this finding could be a false impression and might be caused by an extraordinary increase in number of blood cultures requested during recent years. In Pakistan, laboratory diagnosis of enteric 
fever is commonly made on the basis of either positive serologic test results or blood culture. In view of low sensitivity and specificity of available serologic tests, our clinical laboratory purposely removed these isolates from its test procedure during 2015. Thus, blood culture is currently used as the main tool for diagnosis of enteric fever. During 2017, the number of XDR Salmonella Typhi cases increased from 21 during 2016 to 526 during 2017, but there was no similar emergence of XDR Salmonella Typhi in deep-seated infections.

Whole-genome sequencing of initial outbreak XDR isolates identified plasmid encoding resistance elements, including the $b l a_{\mathrm{CTX}-\mathrm{M}-15}$ extended-spectrum $\beta$-lactamase carrying the qnrS fluoroquinolone resistance gene. This IncY plasmid exhibited high sequence identity to plasmids found in other enteric bacteria isolated from widely distributed geographic locations (13). However, in our study, molecular analysis was not performed, which is a limitation. In addition, our study was retrospective and single-laboratory based. Therefore, our study does not reflect the experience from other centers.

There was no increase in the proportion of XDR Salmonella Typhi extraintestinal isolates compared with non-XDR isolates. Because of the high endemicity of XDR Salmonella Typhi in Pakistan, general practitioners in outpatient clinics usually start empirical treatment for enteric fever on the basis of clinical judgment and serologic investigations. Enteric fever clinical practice guidelines specific to Pakistan are available (14) and should be followed to avoid complications of the disease. Lack of physician awareness regarding extraintestinal seeding of Salmonella Typhi can lead to inappropriate treatment. Thus, culturing of organisms is recommended before starting treatment.

\section{Acknowledgments}

We thank Yusra Shafqat for collecting clinical information, Faisal Malik for collecting laboratory data, and Ghazala Jabeen and Samia Tariq for providing technical support.

\section{About the Author}

Dr. Irfan is a clinical microbiologist and associate professor in the department of pathology and Laboratory Medicine at Aga Khan University, Karachi, Pakistan. Her primary research interests are antimicrobial drug resistance, its laboratory detection methods, testing newer drug options, and infection prevention and control.

\section{References}

1. Mogasale V, Maskery B, Ochiai RL, Lee JS, Mogasale VV, Ramani E, et al. Burden of typhoid fever in low-income and middle-income countries: a systematic, literature-based update with risk-factor adjustment. Lancet Glob Health. 2014;2:e570-80. https://doi.org/10.1016/ S2214-109X(14)70301-8

2. Matono T, Morita M, Yahara K, Lee K-i, Izumiya H, Kaku M, et al. Emergence of resistance mutations in Salmonells enterica serovar Typhi against fluoroquinolones. Open Forum Infect Dis. 2017;4:eof 230.

3. Malik A, Yasar A, Tabinda A, Abubakar M. Water-borne diseases, cost of illness and willingness to pay for diseases interventions in rural communities of developing countries. Iran J Public Health. 2012;41:39-49.

4. Daud MK, Nafees M, Ali S, Rizwan M, Bajwa RA, Shakoor MB, et al. Drinking water quality status and contamination in Pakistan. BioMed Res Int. 2017; 2017:7908183. https:// doi.org/10.1155/2017/7908183

5. Das JK, Hasan R, Zafar A, Ahmed I, Ikram A, Nizamuddin S, et al. Trends, associations, and antimicrobial resistance of Salmonella typhi and paratyphi in Pakistan. Am J Trop Med Hyg. 2018;99(Suppl):48-54. https://doi.org/10.4269/ajtmh.18-0145

6. Huang DB, DuPont HL. Problem pathogens: extra-intestinal complications of Salmonella enterica serotype Typhi infection. Lancet Infect Dis. 2005;5:341-8. https:/ / doi.org/10.1016/ S1473-3099(05)70138-9

7. Rohilla R, Bhatia M, Gupta P, Singh A, Shankar R, Omar BJ. Salmonella osteomyelitis: a rare extraintestinal manifestation of an endemic pathogen. J Lab Physicians. 2019;11:164-70. https://doi.org/10.4103/JLP.JLP_165_18

8. Villablanca P, Mohananey D, Meier G, Yap JE, Chouksey S, Abegunde AT. Salmonella Berta myocarditis: case report and systematic review of non-typhoid Salmonella myocarditis. World J Cardiol. 2015;7:931-7. https://doi.org/10.4330/ wjc.v7.i12.931

9. Meyyur Aravamudan V, Kee Fong P, Singh P, Sze Chin J, Sam YS, Tambyah PA. Extraintestinal salmonellosis in the immunocompromised: an unusual case of pyomyositis. Case Rep Med. 2017;2017:5030961. https://doi.org/10.1155/2017/5030961

10. Mushtaq MA. What after ciprofloxacin and ceftriaxone in treatment of Salmonella Typhi. Pak J Med Sci. 2006;22:51-4.

11. Qamar FN, Azmatullah A, Kazi AM, Khan E, Zaidi AK. A three-year review of antimicrobial resistance of Salmonella enterica serovars Typhi and Paratyphi A in Pakistan. J Infect Dev Ctries. 2014;8:981-6. https://doi.org/10.3855/jidc.3817

12. Crump JA, Sjölund-Karlsson M, Gordon MA, Parry CM. Epidemiology, clinical presentation, laboratory diagnosis, antimicrobial resistance, and antimicrobial management of invasive Salmonella infections. Clin Microbiol Rev. 2015;28:90137. https:/ / doi.org/10.1128/CMR.00002-15

13. Klemm EJ, Shakoor S, Page AJ, Qamar FN, Judge K, Saeed DK, et al. Emergence of an extensively drug-resistant Salmonella enterica serovar Typhi clone harboring a promiscuous plasmid encoding resistance to fluoroquinolones and third-generation cephalosporins. MBio. 2018;9:e00105-18. https://doi.org/10.1128/mBio.00105-18

14. National Institute of Health. Advisory for prevention and treatment of typhoid fever including XDR typhoid, July 2020 [cited 2020 Nov 27]. https:/ / www.nih.org.pk/wp-content/ uploads/2020/07/Advisory-for-Prevention-and-Treatmentof-Typhoid-Fever-including-XDR-Typhoid.pdf

Address for correspondence: Seema Irfan, Section of

Microbiology, Department of Pathology and Laboratory Medicine, Aga Khan University, PO Box 3500, Stadium R, Karachi 74800,

Pakistan; email: seema.irfan@aku.edu 